\title{
P 166 DEVELOPING AN EDUCATIONAL INTERVENTION TO ADDRESS THE DISCREPANCIES IN PRN 'AS REQUIRED' PRESCRIPTIONS WITHIN A HOSPICE SETTING
}

Declan Cawley ${ }^{1,2}$. ${ }^{1}$ Pilgrims Hospices, Canterbury, UK; ${ }^{2}$ University of Kent

10.1136/bmjspcare-2014-000654.207

Background Within the current healthcare arena, the identification of risks within organisations and minimising their negative impact on patient care is fundamental. Reports from the national and international literature have identified strong opioids and sedatives as a cause for concern within clinical practice. Recent baseline data identified significant variations in the practice of prescribing and administration of these drugs even within one organisation.

Aims To develop an educational intervention that is informed by clinical governance practices and utilising current work based assessments to evidence and embed good clinical practice.

Results A baseline review of PRN 'as required' medications identified significant variations in drug dose and escalation, frequency of administration that doesn't match its' pharmacokinetics but importantly inconsistent documentation supporting 'indication or use' to guide nursing administration. A document was produced to highlight the issues from the baseline review, detailing the particular areas of concern and what current guidance suggests. In step 1, clinical vignettes were presented with the view of self-completion prior of Step 2. Within Step 2, trainees gave feedback on their rationale for their prescribing and what they actually documented. The case based discussion approach $(\mathrm{CbD})$ was rated as very useful by trainees new to the specialty. A pre-completed sheet with appropriate prescribing and rationale was given to the trainee with useful resources after the discussion.

Conclusions Within clinical practice, implementation of good practice guidance is fundamental to the success of any audit or baseline review of discrepancies in care. However there is a lack of work based assessments combining guidance, an opportunity for self-completion and then informed feedback so that an individual's learning needs can be appropriately highlighted and subsequently addressed. We believe the principle of this approach has the wider opportunity to tailor the learning needs of the trainee within certain challenging areas of practice. 